Para enlazar con este artículo / To link to this article:

http://dx.doi.org/10.6035/MonTI.2019.ne5.6

Para citar este artículo / To cite this article:

Del Pozo Triviño, Maribel \& Doris Fernandes del Pozo. (2019) "La reputación en línea de la profesión de intérprete en la prensa digital española: el caso de la Voz de Galicia y El País." En: Montero Küpper, Silvia; Montserrat Vázquez Gestal \& Iván Puentes Rivera (eds.) 2019. Comunicación, Traducción e Interpretación / Communication, Translation and Interpreting. MonTI Special Issue 5, pp. 147-166.

\title{
LA REPUTACIÓN EN LÍNEA DE LA PROFESIÓN DE INTÉRPRETE EN LA PRENSA DIGITAL ESPAÑOLA: EL CASO DE LA VOZ DE GALICIA Y EL PAÍS
}

\section{STUDY OF THE ONLINE REPUTATION OF INTERPRETERS IN SPANISH DIGITAL MEDIA: A FIRST APPROACH}

\author{
Maribel Del Pozo Triviño ${ }^{1}$ \\ mdelpozo@uvigo.es \\ Universidade de Vigo \\ DORIS FERNANDES DEL POZO \\ dorisfernandesdelpozo@gmail.com \\ Universidad de Santiago de Compostela
}

\section{Resumen}

El presente trabajo constituye una aproximación a la reputación en línea de la profesión de intérprete a través del análisis de dos medios de prensa digital generalista española con importante difusión en Galicia. A partir de un corpus de noticias de las

1. La autora es miembro titular del grupo de Investigación en Estudos Literarios e Culturais, Tradución e Interpretación BiFeGA, Programa de Consolidación e Estruturación de Unidades de Investigación Competitivas (Ref. ED431B 2017176) de la Universidade de Vigo y de la Rede Internacional de Investigación de Xestión da Comunicación (Ref. ED341D R2016/019 XESCOM) coordinada por la Universidade de Santiago; ambos financiados en régimen de concurrencia competitiva por la Xunta de Galicia. La redacción de este trabajo forma parte de las actividades de investigación promovidas por la Red XESCOM. 
versiones digitales de La Voz de Galicia y El País durante el periodo 2013-2017, se analiza la visibilidad de la interpretación, así como la reputación en línea de las/os intérpretes. Para elaborar el corpus, se han recopilado todas las noticias que hacían referencia directa o indirecta a la interpretación a través de las hemerotecas digitales de los citados diarios. Posteriormente, se ha analizado la visibilidad de cada técnica y modalidad de interpretación, así como la valoración de la figura del/de la intérprete que se hace en el contenido periodístico. Tras el análisis de los resultados, se constata una presencia muy reducida de la profesión de intérprete, así como una valoración mayoritariamente positiva de la misma en los medios analizados.

Palabras clave: Interpretación; Intérprete; Visibilidad de la interpretación; Interpretación en los medios; Reputación en línea

\begin{abstract}
This study is a first approach to ascertaining the online reputation of interpreters in the Spanish digital press through the analysis of two non-specialised online newspapers popular in Galicia. A news corpus from the digital versions of the newspapers "La Voz de Galicia" and "El País" was analysed for the period 2013-2017 to ascertain the visibility of interpretation and the online reputation of interpreters. All news items with direct or indirect reference to interpretation were taken from the digital archive of the said newspapers. The visibility of each interpreting technique and mode were then analysed together with the perception of the interpreter's figure. Results indicate a poor presence of this profession but a highly positive perception of the same in the 2 media subject to analysis.
\end{abstract}

Keywords: Interpreting; Interpreter; Visibility of interpreting; Interpreting for the media; Online reputation

\title{
1. Introducción
}

La interpretación, entendida como mediación lingüística oral entre personas que no comparten un idioma, se ha venido practicando desde la Antigüedad, aunque su reconocimiento como profesión moderna se produjo a partir de la celebración de los juicios de Núremberg, posteriores a la II Guerra Mundial, y a la creación de organismos internacionales multilingües como la ONU o la UE (Baigorri Jalón 2000). El hecho de que sea una profesión nueva implica que su visibilidad en la sociedad sea todavía reducida, que se confunda con otras profesiones cercanas (como la traducción o la mediación intercultural), 
o que exista un altísimo grado de intrusismo profesional en sectores tan sensibles como los servicios públicos (juzgados, comisarías, hospitales, centros educativos, etc.). La figura del profesional de la interpretación, por tanto, no está bien definida en el imaginario colectivo, algo a lo que contribuyen también los hechos que en ciertas ocasiones trascienden a los medios de comunicación. Sin embargo, el estudio de la presencia de la interpretación en los medios ha recibido escasa atención por parte de la academia hasta la fecha, salvo en trabajos que se centran en escenarios concretos, como la visibilidad mediática de la interpretación en zonas de conflicto (Baker 2010), los que analizan la visibilidad en los medios de la traducción y la interpretación en los servicios públicos (TISP) (Campos Freire \& Fernandes del Pozo 2011 y Cedillo Corrochano 2016), o la visibilidad de la interpretación en medios de comunicación digitales (Táboas Domínguez 2017).

\section{La profesión de intérprete: historia y definición}

\subsection{Historia}

El oficio de intérprete nació cuando los diferentes pueblos del mundo se pusieron en contacto y necesitaron comunicarse. Así pues, existen testimonios que reflejan su existencia ya en Egipto, en el año 3000 a.C. donde un relieve de piedra en la tumba de un príncipe de Elefantina muestra la existencia de un intérprete. También el historiador griego Herodoto dejó claro que la interpretación era una actividad profesional más entre los gremios profesionales del Antiguo Egipto; y tenemos noticia de la existencia de intérpretes en otras poblaciones de la Antigüedad, como Grecia o el Imperio Romano, donde oradores como Cicerón ya afirmaban que sólo un mal intérprete traduciría palabra por palabra. Sin embargo, cabe decir que los intérpretes no fueron siempre admirados en la Antigua Roma, ya que a menudo eran esclavos, prisioneros de guerra, etc. y por lo tanto, no se les consideraba como figuras fiables. No será hasta la Edad Media cuando los intérpretes sean admirados por sus habilidades y lleguen a ser incluso miembros de la Corte. En esta época se llevó a cabo la expansión del cristianismo en Europa y esto habría sido imposible sin el gran papel desempeñado por intérpretes monásticos, misioneros, etc. En los tiempos de la Conquista, los exploradores viajaron a América con intérpretes de árabe y hebreo, dándose cuenta al llegar a estas 
tierras desconocidas de que poco les servían estos idiomas (Alonso Araguás \& Baigorri Jalón 2002). Sin embargo, su voluntad por comunicarse con los indígenas los llevó incluso a realizar secuestros para enseñarles el castellano y utilizarlos de intérpretes posteriormente. Siglos más tarde, tuvieron lugar expediciones en África y en Asia donde la interpretación fue crucial. Entretanto, Francia emergió como la principal potencia en Europa, lo que llevo al francés a convertirse en la lengua culta y de la diplomacia. Esto provocó una disminución de la necesidad de intérpretes. No obstante, pese a que los diplomáticos se comunicaban mayoritariamente en francés, el servicio de los intérpretes siguió siendo demandado por emperadores, misioneros, comerciantes, etc. de países que no siguieron esta tendencia.

Como ya se adelantaba en la Introducción, el reconocimiento de la interpretación como profesión moderna empieza a producirse en el periodo entre la I y la II Guerra Mundial y consigue su consolidación definitiva a partir de la celebración de los juicios de Núremberg y de la creación de organismos internacionales multilingües como la ONU o la UE (Baigorri Jalón 2000).

\subsection{Definición}

Como se desprende de los párrafos anteriores, la interpretación ha ido afinando su definición a lo largo de la historia hasta convertirse en un concepto amplio que en la actualidad incluye cuestiones tales como: transferencia cultural, implicaciones pragmáticas, funcionalidad e incluso independencia del texto término como producto válido en sí mismo (Alonso Bacigalupe 1998: 15). Una de las primeras definiciones formales de la labor del intérprete, tal y como la entendemos en la actualidad la ofrece Herbert ya en el año 1952 en su manual sobre la interpretación de conferencias:

The mission of the interpreter is to help individuals and communities to acquire a fuller knowledge and a deeper understanding of one another, and, what is still more important, a greater respect for one another. Also to come to an agreement if they should want to do so (1952: 3).

Con esta definición Herbert se aparta de la idea tradicionalmente mantenida de que el intérprete es una especie de máquina encargada de buscar automática y rápidamente equivalencias en una lengua a las palabras emitidas en otra lengua distinta. Gile (1995) da un paso más en la definición de la profesión 
y resalta ciertos elementos primordiales para comprender qué es la interpretación desde la realidad del acto interpretativo que comprende factores tan importantes como: la función comunicativa, el servicio de comunicación, los entornos de trabajo, los receptores del servicio, los aspectos económicos, las conductas profesionales y éticas, etc. El autor entiende, pues, la interpretación como un servicio que se presta a alguien y cuyo éxito dependerá de muchos factores. Gentile (1996: 39) también retoma la idea de servicio, e incluye, de manera explícita, la mención al contexto situacional y temporal, así como a la dimensión semántica, semiótica y pragmática del intercambio comunicativo:

The essence of interpreting skills is to receive a message which is not formulated for the interpreter and to deliver that message in a form and a character indistinguishable from the original. The message includes all the elements which constitute the act of communication (comprising semantic, pragmatic and semiotic dimensions) in a particular temporal and situational context. The skills of an interpreter therefore extend beyond the linguistic and include the capacity to internalize and transmit the many nuances of a particular situation.

Todas estas definiciones, y muchas otras, pueden ayudarnos a entender un poco mejor qué es la interpretación y, según Alonso (1998: 20), uniendo los diferentes elementos señalados por los autores anteriormente mencionados, podemos llegar a una definición global:

La interpretación es una actividad profesional remunerada -y sujeta, por tanto, a unas normas de comportamiento profesional- consistente en la transferencia de un mensaje de una LO a una LT para un público determinado, en un contexto y situación también específicos, buscando una correspondencia pragmática, semiótica y semántica del mensaje, mediante la comprensión de todos los elementos que lo integran para alcanzar así el objetivo de una comunicación intercultural plena entre individuos de distintas lenguas haciendo uso de las explicaciones necesarias para que esa comunicación sea efectiva.

\section{Justificación: por qué estudiar la reputación en línea de la profesión de intérprete}

A pesar de los avances en la consolidación de la profesión de intérprete, los problemas actuales a los que se enfrenta esta profesión en España son múltiples, y entre ellos destaca especialmente la falta de profesionalización, 
entendida como una falta de reconocimiento social e institucional de la formación superior necesaria para desempeñar el oficio de intérprete. Las causas que subyacen a esta falta de profesionalización han sido objeto de diversos estudios (Ortega Herráez 2010, Del Pozo Triviño 2013, Blasco Mayor \& Del Pozo Triviño 2015, etc.). Dos de los argumentos recurrentes y complementarios son, por un lado, la escasa y difusa proyección social de la profesión, y por otro, el hecho de que la figura moderna del intérprete profesional sea relativamente reciente, y esté en exceso circunscrita al ámbito de la interpretación de conferencias, como le ocurre a la profesión de traductor/a con la traducción literaria.

La falta de profesionalización lleva aparejada otra serie de problemas que afectan a la profesión de intérprete, como es el elevado grado de instrusismo profesional (Monzó Nebot \& Borja Albi 2005). El intrusismo no solo tiene consecuencias negativas para el colectivo de intérpretes profesionales en lo que concierne al acceso al mercado de trabajo, sino que conlleva una merma sustancial de la calidad de la interpretación y ocasiona importantes problemas a las personas usuarias de mediación lingüística. Esto resulta especialmente grave en ámbitos tan sensibles como los servicios públicos (policía, tribunales, sanidad, educación, etc.), donde se da un mayor grado de intrusismo laboral debido a los laxos procedimientos de contratación aplicados por las Administraciones públicas y la ausencia de controles de calidad y/o mecanismos de queja efectivos (Ortega Herráez 2010, Blasco Mayor \& Del Pozo Triviño 2015). La mayor presencia de población extranjera en España, derivada del aumento del turismo, de los intensos procesos migratorios y de la movilidad europea ofrecida dentro del espacio Schengen, hacen que el contacto con personas extranjeras sea cada vez mayor, en especial en hospitales, escuelas, comisarías o juzgados.

A pesar del aumento del contacto con población extranjera, la interpretación no se garantiza en la mayoría de los servicios públicos, y cuando está disponible, a menudo se permite la contratación de personas no cualificadas, en el mejor de los casos. Sin embargo, esta falta de profesionalización e incorporación de la interpretación de calidad a la red de servicios públicos hace que se produzcan situaciones de auténtica vulnerabilidad lingüística o merma de la eficacia del trabajo de profesionales públicos. Llaman la atención especialmente los casos en que se han contratado a personas con antecedentes penales 
para intervenir en investigaciones por terrorismo yihadista ${ }^{2}$, por ejemplo, pero hay muchas otras situaciones dramáticas sin cobertura mediática en que se usa a menores para realizar la interpretación a sus padres (Greene et al. 2005), a los propios maltratadores para comunicarse con víctimas de violencia de género (Del Pozo Triviño et al. 2014), a voluntarios con personas refugiadas y/o solicitantes de asilo (León Pinilla et al. 2016) o a internos que chapurrean la lengua para comunicarse con la población reclusa ${ }^{3}$. Esta situación ha sido denunciada de manera recurrente en nuestro país por las principales asociaciones profesionales, unidas en la Red Vértice ${ }^{4}$, y documentados de manera conclusiva en el ámbito académico, en especial a través de los proyectos y trabajos de investigación de los miembros de la Red Comunica ${ }^{5}$, entre los que cabe destacar el proyecto Traducción e Interpretación en los Procesos Penales $(\text { TIPp })^{6}$ y Speak Out for Support (SOS-VICS) ${ }^{7}$.

La precariedad de la interpretación en los servicios públicos no es un problema exclusivo del contexto español sino que está también presente en países con una riqueza migratoria más variada y antigua, en los que las necesidades de interpretación en los servicios públicos también son muy elevadas. Es el caso, por ejemplo, de Reino Unido, Canadá, Estados Unidos o Australia, donde a

2. Bandera, M. (2008) Interior emplea traductores sin garantías en sus investigaciones (Diario Público): http://www.publico.es/espana/interior-emplea-traductores-garantias-investigaciones.html

3. Peñalosa, Gemma (2008) La falta de traductores impide a la juez de Fontcalent comunicarse con reos extranjeros (El Mundo): http://www.elmundo.es/elmundo/2008/03/22/ valencia/1206194259.html y El Confidencial Digital (2014) El aumento de los reclusos islamistas obliga a Prisiones a contratar traductores de dialectos árabes: https:// www.elconfidencialdigital.com/seguridad/islamistas-reclusos-prisiones-dialectos_ arabes_0_2351764827.html

4. La Red Vértice es una unión de diversas asociaciones profesionales de intérpretes y traductoras/es que ha hecho un seguimiento y se ha pronunciado sobre la precaria situación de la interpretación en los servicios públicos. En su página web pueden leerse diversos comunicados: http://www.redvertice.org/p/comunicados.html

5. La Red Comunica es el Observatorio permanente sobre traducción e interpretación en los servicios públicos (TISP) y está formada por diferentes grupos/proyectos de investigación españoles especializados en la TISP, como lo son el Grupo Alfaqueque (USAL), el grupo MIRAS (UAB) o el proyecto SOS-VICS (Uvigo), entre otros: http:// red-comunica.blogspot.com.es/

6. http://pagines.uab.cat/tipp/

7. http://sosvicsweb.webs.uvigo.es/ 
pesar de contar con una mayor proyección de la figura del/de la intérprete profesional, se siguen documentado y combatiendo casos de vulnerabilidad lingüística por causa de la falta de aptitud del personal contratado para realizar la interpretación.

Por ende, el intrusismo profesional y la resultante fluctuación en la calidad de los servicios de interpretación prestados, tanto en los servicios públicos como en el ámbito privado, afectan a la imagen que se tiene de la interpretación y de las personas que trabajan como intérpretes, aunque en el ámbito privado se da en menor medida. Esto compromete el prestigio social del trabajo de las y los intérpretes, lo que a su vez contribuye a perpetuar la falta de profesionalización efectiva. Sin embargo, a pesar de que esta es una queja extendida en los colectivos profesionales y académicos, hasta el momento no se ha prestado una especial atención a la imagen de la profesión de intérprete, ni a efectos de documentarla ni a efectos de influir sobre ella a través de campañas de comunicación específicas.

De la misma manera, tampoco existen trabajos dedicados a medir ni analizar la presencia en la agenda de los medios de comunicación de la profesión de intérprete, como tampoco hay constancia de trabajos que aborden la presencia en redes sociales. En la actual sociedad de la información, donde el papel de las redes sociales y la comunicación digital cobra cada vez mayor importancia, el seguimiento de la presencia en medios y el análisis de la reputación en línea son cuestiones imprescindibles para abordar la comunicación de una empresa, institución, etc.

Visibility refers to the public presence of an individual or organization in the media, and has an influence on organizational perceptions in times of crisis, buying preferences, and trust (Yang \& Kent 2014: 563).

Hasta la fecha de publicación de este artículo, los únicos trabajos sobre la visibilidad de la interpretación en los medios de los que se tiene constancia son los de Baker (2010), Campos Freire y Fernandes del Pozo (2011), Cedillo Corrochano (2016) y Táboas Domínguez (2017).

Drawing on available studies and recent media reports on contemporary conflicts, and adopting a narrative perspective to make sense of the findings, this article focuses on two issues. The first is how translators and interpreters are narrated by other participants in the war zone, including military personnel, war correspondents, mainstream media, alternative media and local 
populations. The second is how they themselves participate in elaborating the range of public narratives of the conflict that become available to us, and, in so doing, influence the course of the war in ways that are subtle, often invisible, but nevertheless extremely significant (Baker 2010: 197).

Por su parte, los trabajos de Campos Freire y Fernandes del Pozo (2011) y el de Cedillo Corrochano (2016) abordan la presencia de la TISP en los medios digitales de comunicación y en redes sociales. Ambos trabajos, si bien cuentan con una metodología limitada, analizan una amplia muestra y coincidieron en la invisibilidad de la TISP en el panorama mediático. El trabajo de Táboas Domínguez es un trabajo de fin de grado inédito presentado en la Universidad de Vigo en el año 2017 en el que se hace una primera aproximación a la presencia de la interpretación en las informaciones de tres diarios generalistas, con una metodología también limitada, pero que es testimonio del incipiente interés por esta cuestión.

Por todo lo anterior, ante la ausencia de estudios y datos en este ámbito y con el fin de contribuir a realizar un seguimiento de la presencia de la interpretación en los medios de comunicación y de la reputación en línea de la profesión de intérprete, como primer paso para avanzar hacia una mejor gestión de la reputación y la imagen de la profesión de intérprete y contribuir, de este modo, a su profesionalización, presentamos el siguiente trabajo preliminar de investigación sobre la reputación online de la interpretación en dos ejemplos de prensa digital española. Se trataría de un primer paso para avanzar hacia una mejor gestión de la reputación y la imagen de la profesión de intérprete y contribuir, de este modo, a su profesionalización.

\section{La reputación en línea de la profesión de intérprete en la prensa digital española: estudio en La Voz de Galicia y El País (2013-2017)}

El presente trabajo es una primera aproximación a la imagen que los medios de comunicación proyectan de la profesión de intérprete en los medios digitales españoles y, por ende, al estudio de la reputación en línea de la profesión de intérprete en la prensa digital española. La ausencia de datos previos y de trabajos de investigación en esta materia hace que sea difícil acotar la dimensión de un primer estudio de esta naturaleza, lo que a su vez limita el alcance y la representatividad de los resultados. En este trabajo nos proponemos tratar de dibujar un perfil de la situación actual que sirva como primer peldaño 
para continuar explorando de manera más amplia y profunda la reputación en línea de la profesión de intérprete y su evolución.

\subsection{Metodología}

La metodología empleada en este estudio es fundamentalmente cuantitativa. La muestra seleccionada para realizar este trabajo han sido las informaciones recogidas durante un lustro en las versiones electrónicas de dos medios españoles generalistas de referencia: El País y La Voz de Galicia. La razón para escoger estos dos medios es que fueron el segundo y quinto diario en número de lectores según el Estudio General de Medios (AIMC 2017), siendo ambos de difusión nacional, pero uno de carácter nacional y otro de carácter regional (Galicia).

Se ha elaborado un corpus de noticias de las versiones digitales de estos diarios durante el periodo de 2013-2017, mediante la recopilación de aquellas informaciones que hacían referencia directa o indirecta a la interpretación o a las/os intérpretes, lo que arrojó un total de 37 resultados. Para identificar aquellas noticias susceptibles de ser analizadas en el marco de este trabajo se introdujeron las palabras clave "interpretación", "intérprete", "traductor", "traductora" y "traducción" en los motores de búsqueda de las hemerotecas digitales de los citados diarios, ya que en numerosas ocasiones se confunde la actividad de intérprete con la de traductor. De los resultados obtenidos, se filtraron aquellos en que se hacía referencia únicamente a la interpretación lingüística, dejando fuera aquellos resultados relacionados con la traducción (escrita) o con la interpretación entendida como concepto artístico (interpretación/intérprete musical, teatral, de cine, etc.). También se han excluido los resultados hallados en blogs corporativos o piezas de opinión ciudadana, puesto que el objeto de este estudio es conocer la reputación en línea de la profesión de intérprete entendida como el resultado de su visibilidad y tratamiento en los medios digitales de comunicación. Es necesario tener en cuenta que, dado que la búsqueda se ha realizado de manera manual empleando palabras clave, y a pesar del carácter exhaustivo de la misma, es posible que haya resultados que hayan quedado fuera del alcance de este estudio por no estar etiquetados con ninguna de las palabras clave utilizadas. 
Posteriormente, los resultados se recogieron en una tabla individual ${ }^{8}$ para cada diario, asignándoles un código conformado por dos letras (en referencia al nombre del diario del que fueron extraídas) y seguida por una secuencia numérica ordinal correlativa en función de la fecha de publicación. La tabla contiene campos de descripción y de análisis para facilitar el tratamiento de los datos y su análisis posterior. En lo que concierne a los campos de descripción, tras el código (de clasificación) aparece el campo para las palabras clave utilizadas para localizar la pieza informativa en la hemeroteca digital del diario. A continuación, se indican la fecha, la sección y el titular de la pieza periodística. Por último, se indica cuál es la URL de la información. Los campos relacionados con la clasificación vienen tras el titular, en los que se identifica la técnica de interpretacón, la modalidad de interpretación a la que se alude en la información y la valoración que se ofrece a criterio de las autoras (neutra, negativa o positiva).

Con los datos anteriores, hemos realizado un primer análisis para determinar cuál es la presencia y, por ende, la visibilidad de la interpretación en la prensa digital española. A continuación, hemos analizado cuál es el alcance de la información en cuestión y el tratamiento que se le da a la figura del intérprete, con la intención de ofrecer con ello una fotografía inicial de la reputación en línea de las/os intérpretes. El empleo de un enfoque cuantitativo-cualitativo parte de la vocación de análisis de contenido del presente trabajo, en el que un enfoque mixto se hace indispensable para poder llevar a cabo la investigación (Krippendorff 2004: 87).

\subsection{Resultados}

Los resultados de este estudio para el periodo 2013-2017 revelan que se publicaron un total de 37 informaciones relativas a la profesión de intérprete o a la interpretación, 9 de ellas en La Voz de Galicia y 28 en El País. Las informaciones recogidas en La Voz de Galicia se publicaron en la sección de Internacional (3), Deportes (2), Galicia (1) y las 3 restantes en ediciones locales ( 2 en Vigo Ciudad y 1 en Ferrol Ciudad). En cuanto a las piezas periodísticas publicadas en El País, estas se publicaron mayoritariamente

8. Veánse Anexos 1 y 2. 
en las secciones de Internacional (9), España (4) y Sociedad (2), siendo el resto publicadas en otras secciones heterogéneas, como Planeta Futuro (1), Operación Púnica (1), A Fondo (1), En primera línea (1), Babelia (1), Estados Unidos (1), La Crónica (1), Vídeos (1), Cultura (1), así como 2 piezas en ediciones autonómicas (Cataluña 1, Galicia 1) y 2 de ellas sin estar adscritas a ninguna sección en concreto.

A pesar de no contar con el volumen total de noticias publicadas en la versión digital de estos diarios para poder establecer la ratio de noticias sobre interpretación respecto de la agenda de los medios analizados en este estudio, lo cual supone una clara limitación de la metodología, estos resultados nos permiten sugerir que la presencia de la interpretación o de la figura de un/a intérprete en los medios digitales consultados es, cuando menos, escasa. Como cabría esperar por la naturaleza intercultural de la profesión de intérprete, una parte importante de las informaciones publicadas en ambos diarios se encuentra en la sección de Internacional, pero resulta llamativo que el resto de informaciones aparezcan publicadas en secciones muy diversas, lo que podría interpretarse como una aparición casi anecdótica de la interpretación.

En lo que atañe a la visibilidad de las diferentes técnicas de interpretación, la más visible es la interpretación de enlace (28 informaciones), puesto que solo hubo 2 informaciones de El País que aludieron de manera explícita a la interpretación simultánea y a la transcripción de escuchas telefónicas. El resto de informaciones (7) no hacían referencia a la técnica, y en ninguna se hizo mención a la interpretación consecutiva.

Es conveniente interpretar los resultados relacionados con las técnicas con los relativos a las modalidades de interpretación, puesto que a través de las modalidades también es posible inferir a qué técnica se hacía referencia. La modalidad más mencionada fue la intepretación en los servicios públicos (15), seguida por la interpretación militar o en zonas de conflicto (11) y la interpretación de conferencias (4), y en menor medida, la interpretación diplomática (3), la interpretación en la historia (2), en los deportes (1) o en los medios de comunicación (1). El hecho de que la interpretación de enlace sea la técnica más visible concuerda con el hecho de que la mayor parte de las informaciones hablen de la modalidad de interpretación en los servicios públicos y de la interpretación militar, y aunque la técnica de simultánea solo 
aparezca mencionada una vez, vemos cómo la interpretación de conferencias es la siguiente modalidad más visible.

Una vez obtenidos los resultados relativos a la visibilidad, lo interesante para el abordaje de la reputación en línea de la profesión de intérprete era obtener una idea sobre cuál es la valoración que se hace sobre la interpretación o la figura del intérprete en las informaciones anteriormente citadas. Para ello, tal y como se indica en la metodología, se procedió a realizar la lectura del contenido completo de la pieza informativa que permitiera determinar si la valoración de la interpretación/profesión de intérprete era positiva, negativa o neutra, aunque conviene tener en mente que puede existir un sesgo involuntario en la valoración realizada por las autoras. En algunos casos, por ejemplo, a pesar de la ausencia de un juicio de valor explícito acerca del profesional o ejercicio de la interpretación en la información en cuestión, se han interpretado como «positivas» aquellas noticias en las que se interpreta que la ausencia de interpretación contribuyó a una vulneración de derechos. Claros ejemplos son las noticias EP12 en la que el propio titula ya explica que la ausencia de intéprete causó un grave perjuicio: "Una madre argelina firmó en la Xunta una adopción sin intérprete", y la noticia EP14 titulada "Deportados sin entender nada".

La valoración global obtenida fue de 21 informaciones «positivas», 9 informaciones «neutras» y 7 informaciones «negativas», datos que resultan sorprendentes por la importante presencia de informaciones «positivas» habida cuenta de que no existen, a priori, medidas de comunicación encaminadas a trabajar sobre la reputación de la profesión de intérprete por parte de los colectivos profesionales. Al desagregar estos datos por medios, para La Voz de Galicia se contabilizaron 4 informaciones «neutras», 3 «positivas» y 2 «negativas», mientras que para El País se computaron 18 informaciones «positivas», 5 informaciones «neutras» y 5 «negativas». Aunque existe una ligera variación entre medios, lo cierto es que, a efectos de interpretar los datos, el grueso de las informaciones son o bien «positivas» o «neutras», siendo las noticias «negativas» las menos numerosas ( La Voz de Galicia) o iguales en presencia que las «neutras» (El País). 
Cuadro. Resumen de resultados

-Total de noticias encontradas: 37

-La Voz de Galicia: 9

-El País: 28

-Técnicas:

Simultánea: 1

Consecutiva: 0

Enlace: 28

Transcripción escuchas telefónicas: 1

Sin determinar: 7

-Modalidades:

Militar: 11

Historia: 2

Servicios públicos: 15

Deportes: 1

Medios de comunicación: 1

Diplomática: 3

Conferencias: 4

-Valoración global:

-Neutras: 9

-Positivas: 21

-Negativas: 7

Fuente: Elaboración propia

\subsection{Discusión de los resultados}

Sin perjuicio de la cautela necesaria con la que han de utilizarse los resultados del presente estudio preliminar, que cuenta con las limitaciones metodológicas anteriormente expuestas, este análisis de los resultados obtenidos para el periodo y los medios analizados nos indica que existe una presencia, cuando menos, escasa de la profesión de intérprete en los medios digitales (37 noticias en un periodo de 5 años). El análisis de los resultados también nos sugiere una mayor presencia en la prensa nacional que en la prensa gallega, una mayor visibilidad de la técnica de interpretación de enlace y de la modalidad de interpretación en los servicios públicos, y una mayor presencia de informaciones positivas.

Si bien los resultados confirman que la valoración global de la profesión de intérprete es positiva, la baja visibilidad y el carácter heterogéneo de las 
informaciones (tanto en géneros periodísticos, que no han sido tratados en el presente artículo pero que son variados, como en las secciones en las que aparecen) nos llevan a la conclusión de que la reputación en línea de la interpretación es prácticamente inexistente. Las autoras consideran que sería de gran interés cruzar los resultados del presente estudio con otros trabajos realizados sobre la visibilidad y reputación de la traducción en los medios de comunicación (Montero Küpper y Luna Alonso 2018), puesto que, dada la habitual confusión entre interpretación y traducción, es posible que los datos para el ámbito de la traducción puedan ayudar a enriquecer e interpretar con mayor rigor la radiografía sobre la reputación en línea de la profesión de intérprete.

\section{Conclusión}

La potencial utilidad de estos resultados preliminares, más allá de ofrecernos una radiografía sobre la (casi inexistente) reputación en línea de la profesión de intérprete, pueden servir como evaluación diagnóstica para una posterior estrategia de comunicación coordinada entre los colectivos profesionales que permita construir una presencia más consolidada de la interpretación como profesión liberal contemporánea y reforzar los valores positivos asociados a la misma.

Para poder continuar el estudio iniciado en este trabajo, es necesario ampliar el corpus a otros medios de comunicación generalistas digitales que mejoren la calidad de la muestra. Además de ampliar el corpus será preciso contar con una metodología de compilación más eficiente que la búsqueda manual en los buscadores propios, o al menos contar con la garantía de que los resultados ofrecidos por los buscadores son exhaustivos. Un corpus más amplio permitirá, entre otras cosas, analizar si existen diferencias entre la valoración realizada en las informaciones de diferentes diarios. Sin embargo, la ampliación del corpus exigirá también el perfeccionamiento de las variables empleadas para determinar la valoración (positiva, negativa y neutra), puesto que a medida que aumente el tamaño de la muestra, el análisis y valoración de las informaciones será cada vez más complejo. Asimismo, será necesario que estas variables se ajusten en mayor medida a las variables necesarias para realizar el seguimiento de la reputación en línea. 
Otro aspecto que resulta de interés para el estudio de la reputación en línea de la profesión de intérprete es el análisis del contenido de las informaciones recogidas más allá de la valoración ofrecida, como, por ejemplo, el posicionamiento de la interpretación como tema central, secundario o anecdótico de la información, o su aparición en el titular o encabezado de la información. De acuerdo con el análisis del contenido, especialmente en aquello que se refiere al estudio de la visibilidad de las diferentes técnicas y modalidades, resultará interesante investigar cuáles son los temas con los que se suele relacionar la interpretación (derechos, vulneración de derechos, diversidad, racismo, xenofobia, política, etc.), para lo que se considera necesaria la incorporación del análisis de otras disciplinas académicas a la investigación de la visibilidad de la interpretación (estudios de comunicación, relaciones públicas, publicidad, sociología, análisis del discurso, etc.).

\section{Referencias bibliográficas}

AIMC (Asociación para la investigación de Medios de Comunicación). (2017) Resumen general de octubre 2016 a mayo 2017. Versión electrónica: <http:// www.aimc.es/almc-c0nt3nt/uploads/2017/07/resumegm217.pdf>

AlONSO ARAguÁs, Icíar \& Jesús Baigorri Jalón. (2002) "La mediación lingüístico-cultural en las Crónicas de la Conquista: reflexiones metodológicas en torno a Bernal Díaz del Castillo." Actas del VI Congreso Internacional de Antropología Iberoamericana «Cronistas de Indias». Salamanca: Universidad de Salamanca, pp. 159-165. Versión electrónica: <https://gredos.usal.es/jspui/ bitstream/10366/76636/1/DTI_AlonsoAraguasI_CronicasdelaConquista.pdf> Alonso Bacigalupe, Luís. (1998) Proyecto docente. Técnicas de Interpretación consecutiva. Técnicas de Interpretación simultánea. Universidade de Vigo. Proyecto docente inédito.

BAIGORRI JALÓN, Jesús. (2000) La interpretación de conferencias: el nacimiento de una profesión. De París a Nuremberg. Granada. Comares.

BAKER, Mona. (2010) "Interpreters and Translators in the War Zone." The Translator 16:2, pp. 197-222. DOI: 10.1080/13556509.2010.10799469.

Blasco Mayor, María Jesús \& Maribel del Pozo Treviño. (eds.) 2015. La interpretación judicial en un momento de cambio. MonTI 7.

CAmpos Freire, Francisco \& M $M^{a}$ Dolores Fernandes del Pozo. (2011) "Presencia de la Traducción e Interpretación en los Servicios Públicos en Internet: 
presentación de un nuevo sistema de gestión y seguimiento del contenido online y su aplicación a la TISP.” En: Valero, Carmen (ed.) 2011. Traducción e Interpretación en los Servicios Públicos en un mundo INTERcoNEcTado [DVD]. Alcalá de Henares: Servicio de Publicaciones de la Universidad de Alcalá. Versión electrónica: <http://www3.uah.es/master-tisp-uah/wp-content/ uploads/2015/11/TISP-INTERCONECTADO-2011.pdf\#page=41>

Cedillo Corrochano, Carmen. (2016) "Análisis de la presencia mediática de la TISP en España y de su posible profesionalización.” FITISPos International Journal 3, pp. 164-173. Versión electrónica: <https://ebuah.uah.es/dspace/ bitstream/handle/10017/29641/analisis_cedillo_FITISPos_2016_n.\%203. pdf? sequence $=1$ \&isAllowed $=y>$

Del Pozo TriviÑo, Maribel. (2013) "El camino hacia la profesionalización de los intérpretes en los servicios públicos y asistenciales españoles en el siglo XXI." En: GALA, Candelas Claudia ANGELELLI (eds.) 2013. Cuidado con los vacíos: la universidad y los estudios de traducción e interpretación/Minding the gaps: translation and interpretation studies in academia. Cuadernos de ALDEEU, Volumen 25, Publicación de la Asociación de Licenciados y Doctores Españoles de Estados Unidos, pp: 109-131. Versión electrónica: <http://aldeeu.org/cuadernos/index.php/CALDEEEU/article/viewFile/37/29>

Del Pozo Triviño, Maribel; Antonio Vaamonde Liste; David Casado-Neira; Silvia Pérez Freire; Alba Vaamonde Paniagua; Doris Fernandes del Pozo \& Rut Guinarte Mencía. (2014) Comunicación entre profesionales de la atención en violencia de género y víctimas/supervivientes que no hablan el idioma. Informe sobre la encuesta a agentes del proyecto Speak Out for Support (SOS-VICS). Vigo: Servizo de Publicacións da Universidade de Vigo.

GENTILE, Adolfo. (1996) "Translation Theory Teaching: Connecting Theory and Practice." En: Dollerup, Cay \& Vibeke Appel (eds.) Teaching Translation and Interpreting 3. Amsterdam \& Philadelphia: John Benjamins Publishing Company, pp. 55-63.

GILE, Daniel. (1995) Basic Concepts and Models for Interpreter and Translator Training. Amsterdam \& Philadelphia. John Benjamins Publishing Company. GreEn, Judith; Caroline Free; Vanita Bhavna \& Tony Newmand. (2005) "Translators and mediators: bilingual young people's accounts of their interpreting work in health care." Social Science E Medicine 60:9, pp. 2097-2110. Versión electrónica: <https://www.sciencedirect.com/science/article/pii/ S0277953604004885> 
HERBERT, JEAN. (1952) The Interpreter's Handbook: How to become a conference interpreter. Ginebra: Librairie de L'Université Georg.

KRIPPENDORFF, KLAUS. (2004) Content analysis: an introduction to its methodology, 2nd ed. Londres: Sage Publications.

LEÓN-PINILLA, Ruth; Eivor Jordà-Mathiasen \& Vicente Prado-Gascó, Vicente. (2016) "La interpretación en el contexto de los refugiados: valoración por los agentes implicados." Sendebar 27, pp. 25-49. Versión electrónica: <http:// revistaseug.ugr.es/index.php/sendebar/article/viewFile/4921/5051>

MONTERO KÜPPER, SILVIA \& Ana Luna. (2018) O discurso metatradutivo na prensa española (2013-2017) (inédito), presentado en III XESCOM, Simposio Internacional sobre Gestión de la Comunicación.

Monzó NeBot, Esther \& Anabel Borja Albi. (eds.) (2005) La traducción y la interpretación en las relaciones jurídicas internacionales. Castellón de la Plana: Publicaciones de la Universitat Jaume I.

Ortega Herráez, Juan Miguel. (2010) Interpretar para la justicia. Granada. Comares.

TÁBOAS Domínguez, David. (2017) Análisis sobre la presencia de la interpretación en los tres medios de comunicación. Trabajo de fin de grado inédito: Universidad de Vigo.

YANG, Aimei \& Michael Kent. (2014) "Social media and organizational visibility: A sample of Fortune 500 corporations." Public Relations Review 40, pp. 562-564. http://dx.doi.org/10.1016/j.pubrev.2014.04.006.

\section{NOTA BIOGRÁFICA / BIONOTE}

Maribel Del Pozo TRIViÑo es Doctora en Traducción e Interpretación y Traductora e Intérprete Jurada inglés-español. Ha desarrollado una intensa carrera profesional como traductora e intérprete y actualmente es profesora en la Facultad de Filología y Traducción de la Universidade de Vigo. Tiene numerosas publicaciones relacionadas con la traducción jurídica y TISP y participa en varios proyectos de investigación relacionados con estos ámbitos. Ha sido coordinadora del proyecto europeo Speak Out for Support (SOS-VICS), centrado en la formación de intérpretes para trabajar con víctimas de violencia de género. Ha impartido docencia en numerosas universidades y otras instituciones en España y en el extranjero en numerosos congresos científicos, en 
algunos como ponente invitada. Forma parte de diversas redes asociaciones académicas y profesionales (Red XESCOM, AIETI, Red Comunica, TREC, GENTT, BIFEGA, APTIJ, AGPTI, etc.).

MARIBEL Del POZO TRIVIÑo has a PhD in Translation and Interpretation. She is is a sworn English-Spanish translator and interpreter and has worked as a T\&I for many years. She is currently an assistant professor at the Faculty of Philology \& Translation of the University of Vigo. She has published extensively on different aspects of legal translation and public services translation $\&$ interpreting and is currently involved in several research projects in the mentioned fields. She has coordinated the EU project Speak Out for Support (SOS-VICS) focused on training interpreters to work with victims of gender violence. Besides the above, she has lectured at several universities and institutions both in Spain and abroad, and has participated in several scientific conferences, in some as guest speaker. She is a member of several academic and professional associations (XESCOM, AIETI, Red COMUNICA, TREC, GENTT, BIFEGA, APTIJ, AGPTI, etc.).

Doris Fernandes Del Pozo es Licenciada en Periodismo (USC, 2011), Máster en Comunicación e Industrias Creativas (USC, 2012) y Licenciada en Traducción e Interpretación (UVigo, 2013). Fue asistente de investigación del proyecto europeo Speak Out for Support (SOS-VICS), financiado por el Programa de Justicia Penal de la Comisión Europea (2012-2014), donde trabajó en la elaboración de un programa de formación de intérpretes en el contexto de la atención lingüística a víctimas de violencia de género. Realizó prácticas como Gestora de Comunicación en el Parlamento Europeo (Luxemburgo, 2017) y ha impartido docencia universitaria en traducción jurídica (UVigo, 2017-2018). Actualmente trabaja como intérprete y traductora especializada en textos de la Unión Europea y está realizando su tesis doctoral.

DORIS FERNANDES DEL POZO has a Bachelor's Degree in Journalism (University of Santiago de Compostela, 2011), a Master's Degree in Communication and Creative Industries (University of Santiago de Compostela, 2012), and a Bachelor's Degree in Translation and Interpreting (University of Vigo, 2013). She worked as research assistant in the Speak Out for Support (SOS-VICS) project (funded by the Criminal Justice Programme of the European Union, 2012-2014) in which she contributed to preparing a training program for 
interpreters working with gender violence victims. Her stays abroad include one as a Communications Management trainee at the European Parliament (Luxembourg, 2017). Doris is currently doing her PhD in Journalism, has taught Legal Translation at the University of Vigo, Spain (2017-18) and is currently working as an interpreter and translator (EN-ES), specialising in EU texts. 